\title{
Convergence presque sûre de moyennes de sommes de Riemann
}

\author{
par \\ JEAN-JACQUES RuCH (Strasbourg)
}

Définissons pour tout entier naturel $n=1,2, \ldots$ l'opérateur somme de Riemann d'ordre $n$ associé à une fonction $f$ sur le tore $\mathbb{T}=[0,1[=\mathbb{R} \backslash \mathbb{Z}$, comme suit :

$$
\forall x \in \mathbb{T}, \quad R_{n}(f)(x)=\frac{1}{n} \sum_{0 \leq j<n} f\left(x+\frac{j}{n}\right) .
$$

J. Bourgain (voir $[\mathrm{Bo}]$ ) a prouvé que $\left\{R_{n}(f): n \geq 1\right\}$ a une densité logarithmique, id est :

$$
\forall f \in L^{2}(\mathbb{T}), \quad \frac{1}{\log N} \sum_{n=1}^{N} \frac{1}{n} R_{n}(f) \rightarrow \int_{\mathbb{T}} f d m \quad \text { presque sûrement. }
$$

Nous nous sommes inspirés de sa méthode pour prouver des résultats nouveaux concernant la convergence presque sûre des moyennes habituelles des sommes de Riemann suivant des sous-suites de la suite des entiers. Par contre nous ne savons pas si ce résultat reste vrai pour toute la suite des entiers. Ceci nous paraît être un problème intéressant mais difficile à résoudre.

ThÉorÈme. Soient $0<\varepsilon_{1}<1$ et $\varepsilon_{2} \geq \varepsilon_{1}$ deux réels fixés. Alors pour toute suite d'entiers $\left(N_{s}\right)_{s \geq 1}$ tels que

$$
\forall s \geq 1, \quad N_{s}^{1+\varepsilon_{1}}<N_{s+1}<N_{s}^{1+\varepsilon_{2}},
$$

pour toute fonction $f \in L^{2}(\mathbb{T})$,

$$
A_{N_{s}}(f)=\frac{1}{N_{s}} \sum_{n=1}^{N_{s}} R_{n}(f) \text { converge presque sûrement. }
$$

Démonstration. Considérons deux réels $\varepsilon_{1}$ et $\varepsilon_{2}$ fixés tels que $0<$ $\varepsilon_{1}<1$ et $\varepsilon_{2} \geq \varepsilon_{1}$, et $\left(N_{s}\right)_{s \geq 1}$ une suite d'entiers vérifiant (2).

1991 Mathematics Subject Classification: Primary 60F99; Secondary 28D99.

Key words and phrases: Riemann sums, almost everywhere convergence. 
Notons $e_{k}$ le caractère d'ordre $k$. Un simple calcul montre que

$$
\forall n \geq 1, \forall k \in \mathbb{Z}, \quad R_{n}\left(e_{k}\right)=\delta_{n \mid k} e_{k}, \quad \text { avec } \quad \delta_{n \mid k}= \begin{cases}1 & \text { si } n \mid k, \\ 0 & \text { sinon. }\end{cases}
$$

On obtient alors

$$
\forall s \geq 1, \forall k \in \mathbb{Z}, \quad \frac{1}{N_{s}} \sum_{n=1}^{N_{s}} R_{n}\left(e_{k}\right)=\frac{1}{N_{s}} \sum_{n=1}^{N_{s}} \delta_{n \mid k} e_{k},
$$

de sorte que

$$
\forall k \in \mathbb{Z}, \quad\left|A_{N_{s}}\right| \leq \frac{1}{N_{s}} \sum_{n=1}^{N_{s}} \delta_{n \mid k} \leq \frac{k}{N_{s}} \rightarrow 0, \quad \text { quand } s \rightarrow \infty .
$$

On a donc convergence presque sûre des moyennes $\left\{A_{N_{s}}\left(e_{k}\right): s \geq 1\right\}$ pour tout $k$ dans $\mathbb{Z}$, et donc aussi pour toute combinaison linéaire finie de caractères. Or les combinaisons linéaires finies de caractères forment un sousensemble dense de $L^{2}(\mathbb{T})$, il nous suffit donc de montrer la fermeture de l'ensemble de convergence pour démontrer le théorème. Pour cela nous allons prouver l'inégalité maximale suivante :

$$
\forall f \in L^{2}, \quad\left\|\sup _{s \geq 1}\left|A_{N_{s}}(f)\right|\right\|_{2} \leq C\|f\|_{2} .
$$

Observons tout d'abord que si $f \sim \sum \widehat{f}(k) e_{k}$ alors

$$
\forall n \geq 1, \quad R_{n}(f)=\sum \widehat{f}(k) \delta_{n \mid k} e_{k}=\sum_{n \mid k} \widehat{f}(k) e_{k},
$$

et donc

$$
\forall N \geq 1, \quad \frac{1}{N} \sum_{n=1}^{N} R_{n}(f)=\sum_{k=-\infty}^{\infty} \frac{d(k, N)}{N} \widehat{f}(k) e_{k},
$$

avec $d(k, N)=\operatorname{Card}\{1 \leq n \leq N: n \mid k\}$.

La difficulté lors de l'étude des moyennes réside dans l'estimation des quantités $d(k, N)$. Dans notre démonstration nous remplacerons cette suite de multiplicateurs de Fourier par une autre plus facilement manipulable.

Soient $\chi_{z}$ la fonction indicatrice de $z \mathbb{Z}$, c'est-à-dire,

$$
\forall z \in \mathbb{Z}, \forall n \in \mathbb{Z}, \quad \chi_{z}(n)= \begin{cases}1 & \text { si } z \mid n, \\ 0 & \text { sinon. }\end{cases}
$$

Notons encore

$$
\forall j \geq 1, \quad \mathcal{P}^{j}=\left\{p^{j}: p \text { premier }\right\}, \quad \mathcal{P}^{*}=\bigcup_{j \geq 1} \mathcal{P}^{j} .
$$

Remarquons que pour tous $k \in \mathbb{Z}, N \geq 1$ et $n \in[1, N]$,

$$
\left\{\begin{array}{l}
n \mid k \Leftrightarrow \forall z \in \mathcal{P}^{*}, z \nmid k, \chi_{z}(n)=0, \\
n \nmid k \Leftrightarrow \exists z \in \mathcal{P}^{*}, z \nmid k, \chi_{z}(n)=1,
\end{array}\right.
$$


de sorte que pour tous $N \geq 1$ et $k \in \mathbb{Z}$,

$$
\begin{aligned}
\frac{d(k, N)}{N} & =\frac{1}{N} \sum_{n=1}^{N} \prod_{z \in \mathcal{P}^{*}, z \nmid k}\left(1-\chi_{z}(n)\right) \\
& =\frac{1}{N} \sum_{n=1}^{N} \prod_{z \in \mathcal{P}^{*}, z \nmid k, z \leq N}\left(1-\chi_{z}(n)\right) .
\end{aligned}
$$

Soit de plus $\mu_{z}$ la mesure de probabilité sur le tore définie par

$$
\mu_{z} \equiv\left(1-\frac{1}{z}\right)^{1 / 2} \delta_{0}+\frac{1}{z}\left(1-\left(1-\frac{1}{z}\right)^{1 / 2}\right)\left(\delta_{0}+\delta_{1 / z}+\ldots+\delta_{(z-1) / z}\right)
$$

où $\delta_{i}$ est la mesure de Dirac au point $i$. Lorsqu'on calcule les coefficients de Fourier associés à cette mesure on obtient

$$
\forall k \in \mathbb{Z}, \quad\left|\widehat{\mu}_{z}(k)\right|^{2}=1-\frac{1}{z}+\frac{1}{z} \chi_{z}(k) .
$$

On définit alors une suite de multiplicateurs de Fourier de la façon suivante : pour $N \geq 1$ et $k \in \mathbb{Z}$,

$$
\begin{aligned}
\mu_{k}^{(N)} & =\prod_{z \in \mathcal{P}^{*}, z \leq N}\left|\widehat{\mu}_{z}(k)\right|^{2}=\prod_{z \in \mathcal{P}^{*}, z \leq N}\left(1-\frac{1}{z}+\frac{1}{z} \chi_{z}(k)\right) \\
& =\prod_{z \in \mathcal{P}^{*}, z \leq N, z \nmid k}\left(1-\frac{1}{z}\right) .
\end{aligned}
$$

Soient maintenant

$$
M f=\sup _{s \geq 1}\left|\sum_{k \in \mathbb{Z}} \frac{d\left(k, N_{s}\right)}{N_{s}} \widehat{f}(k) e_{k}\right| \quad \text { et } \quad M_{1} f=\sup _{s \geq 1}\left|\sum_{k \in \mathbb{Z}} \mu_{k}^{\left(N_{s}\right)} \widehat{f}(k) e_{k}\right| .
$$

Pour tout $k \in \mathbb{Z}$ et pour tout $s \geq 1$ on a

$$
\begin{aligned}
\mid \sum_{k \in \mathbb{Z}} \frac{d\left(k, N_{s}\right)}{N_{s}} & \widehat{f}(k) e_{k} \mid \\
& \leq\left|\sum_{k \in \mathbb{Z}} \mu_{k}^{\left(N_{s}\right)} \widehat{f}(k) e_{k}\right|+\left|\sum_{k \in \mathbb{Z}}\left(\frac{d\left(k, N_{s}\right)}{N_{s}}-\mu_{k}^{\left(N_{s}\right)}\right) \widehat{f}(k) e_{k}\right| \\
& \leq M_{1} f+\sup _{s \geq 1}\left|\sum_{k \in \mathbb{Z}}\left(\frac{d\left(k, N_{s}\right)}{N_{s}}-\mu_{k}^{\left(N_{s}\right)}\right) \widehat{f}(k) e_{k}\right| .
\end{aligned}
$$

Donc

$$
M f \leq M_{1} f+\left(\sum_{s \geq 1}\left|\sum_{k \in \mathbb{Z}}\left(\frac{d\left(k, N_{s}\right)}{N_{s}}-\mu_{k}^{\left(N_{s}\right)}\right) \widehat{f}(k) e_{k}\right|^{2}\right)^{1 / 2} .
$$


D'où en intégrant cette inégalité et en appliquant le théorème de Fubini, on obtient pour toute fonction $f$ de $L^{2}$,

$$
\|M f\|_{2} \leq\left\|M_{1} f\right\|_{2}+\sup _{k \in \mathbb{Z}}\left(\sum_{s \geq 1}\left|\frac{d\left(k, N_{s}\right)}{N_{s}}-\mu_{k}^{\left(N_{s}\right)}\right|^{2}\right)^{1 / 2}\|f\|_{2} .
$$

Il nous suffit donc de contrôler les deux quantités suivantes :

$$
\left\|M_{1} f\right\|_{2} \quad \text { et } \sup _{k \in \mathbb{Z}}\left(\sum_{s \geq 1}\left|\frac{d\left(k, N_{s}\right)}{N_{s}}-\mu_{k}^{\left(N_{s}\right)}\right|^{2}\right)^{1 / 2},
$$

pour obtenir la relation (4).

Commençons par majorer $\left\|M_{1} f\right\|_{2}$. Pour cela nous utilisons un théorème du à G. C. Rota dont on peut trouver la démonstration dans [Ro].

ThÉORÈme DE RotA. Soient $\left(T_{n}\right)_{n \geq 1}$ une suite d'opérateurs positifs qui sont des contractions de $L^{1}$ et $L^{\infty}$, et qui laissent les fonctions constantes invariantes. Alors la suite d'opérateurs $T_{1} \ldots T_{n} T_{n}^{*} \ldots T_{1}^{*}$, où $T_{n}^{*}$ est l'opérateur adjoint de $T_{n}$, admet un opérateur maximal borné dans $L^{p}$, $p>1$. Si de plus les $T_{n}$ sont définis par des convolutions sur le tore d'une mesure de probabilité $\mu_{n}$ alors pour $p>1$ on a

$$
\left\|\left.\sup _{n}\left|\sum_{k \in \mathbb{Z}} \prod_{j=1}^{n}\right| \widehat{\mu}_{j}(k)\right|^{2} \widehat{f}(k) e_{k} \mid\right\|_{p} \leq C\|f\|_{p} .
$$

En appliquant la deuxième partie de ce théorème pour $p=2$ on obtient

$$
\left\|M_{1} f\right\|_{2} \leq C\|f\|_{2} \text {. }
$$

Estimons maintenant

$$
\sup _{k \in \mathbb{Z}}\left(\sum_{s \geq 1}\left|\frac{d\left(k, N_{s}\right)}{N_{s}}-\mu_{k}^{\left(N_{s}\right)}\right|^{2}\right)^{1 / 2} .
$$

Nous allons montrer que

$$
\sum_{s \geq 1}\left|\frac{d\left(k, N_{s}\right)}{N_{s}}-\mu_{k}^{\left(N_{s}\right)}\right|^{2}<C
$$

uniformément suivant les valeurs de $k$. Comme

$$
\forall N \geq 1, \forall k \in \mathbb{Z}, \quad\left|\frac{d(k, N)}{N}\right| \leq 1, \quad\left|\mu_{k}^{(N)}\right| \leq 1,
$$

il suffit de montrer que

$$
\sum_{s>r}\left|\frac{d\left(k, N_{s}\right)}{N_{s}}-\mu_{k}^{\left(N_{s}\right)}\right|^{2}<C
$$

pour $r \geq 1$ donné. 
Tout au long du reste de la démonstration nous allons utiliser le lemme suivant, qui est une version non probabiliste du théorème de Poincaré. Alors

Lemme A. Soient $J \geq 1$ et $\left(a_{j}\right)_{1 \leq j \leq J}$ une suite de nombres dans $[0,1]$.

$$
\forall k \geq 1, \quad\left|\prod_{j=1}^{J}\left(1-a_{j}\right)-\sum_{|S|<k}(-1)^{|S|} \prod_{j \in S} a_{j}\right| \leq \sum_{|S|=k} \prod_{j \in S} a_{j},
$$

avec les conventions suivantes :

- si $k=1$,

$$
\sum_{|S|<1}(-1)^{|S|} \prod_{j \in S} a_{j}=1
$$

- si $k \geq J+1$,

$$
\sum_{J+1 \leq|S|<k}(-1)^{|S|} \prod_{j \in S} a_{j}=0, \quad \sum_{|S|=k} \prod_{j \in S} a_{j}=0 .
$$

Preuve. Ce lemme se montre par récurrence sur $J$. Vérifions que le résultat soit vrai pour $J=1$. Si $k=1$ alors

$$
\left|\left(1-a_{1}\right)-\sum_{|S|<1}(-1)^{|S|} \prod_{j \in S} a_{j}\right|=\left|\left(1-a_{1}\right)-1\right|=a_{1} .
$$

Si $k \geq 2$ alors

$$
\left|\left(1-a_{1}\right)-\sum_{|S|<k}(-1)^{|S|} \prod_{j \in S} a_{j}\right|=\left|\left(1-a_{1}\right)-\left(1-a_{1}\right)\right|=0 .
$$

Le lemme est donc vrai pour $J=1$.

Supposons maintenant qu'il soit vrai pour $J \geq 1$, et montrons qu'il l'est encore pour $J+1$.

Si $1 \leq k \leq J$ alors

$$
\begin{aligned}
\mid \prod_{j=1}^{J+1}\left(1-a_{j}\right)- & \sum_{|S|<k}(-1)^{|S|} \prod_{j \in S} a_{j} \mid \\
= & \mid\left(1-a_{J+1}\right) \prod_{j=1}^{J}\left(1-a_{j}\right)-\sum_{|S|<k}(-1)^{|S|} \prod_{j \in S, J+1 \notin S} a_{j} \\
& +a_{J+1} \sum_{|S|<k-1}(-1)^{|S|} \prod_{j \in S, J+1 \notin S} a_{j} \mid
\end{aligned}
$$




$$
\begin{aligned}
\leq & \left|\prod_{j=1}^{J}\left(1-a_{j}\right)-\sum_{|S|<k}(-1)^{|S|} \prod_{j \in S, J+1 \notin S} a_{j}\right| \\
& +a_{J+1}\left|\prod_{j=1}^{J}\left(1-a_{j}\right)-\sum_{|S|<k-1}(-1)^{|S|} \prod_{j \in S, J+1 \notin S} a_{j}\right| \\
\leq & \sum_{|S|=k} \prod_{j \in S, J+1 \notin S} a_{j}+a_{J+1} \sum_{|S|=k-1} \prod_{j \in S, J+1 \notin S} a_{j} \quad \text { (par récurrence) } \\
\leq & \sum_{|S|=k} \prod_{j \in S} a_{j} .
\end{aligned}
$$

Si $k=J+1$ alors

$$
\left|\prod_{j=1}^{J+1}\left(1-a_{j}\right)-\sum_{|S|<k}(-1)^{|S|} \prod_{j \in S} a_{j}\right|=\prod_{j=1}^{J+1} a_{j} .
$$

Si $k \geq J+2$ alors

$$
\left|\prod_{j=1}^{J+1}\left(1-a_{j}\right)-\sum_{|S|<k}(-1)^{|S|} \prod_{j \in S} a_{j}\right|=0 .
$$

On en déduit que le résultat est encore vrai au rang $J+1$. Il est donc vrai pour tout $J \geq 1$.

Nous allons commencer par montrer une première majoration de la quantité $d(k, N) / N$ que l'on a isolé dans le lemme suivant.

Lemme B. Soit

$$
r=\left[4-2 \frac{\log \varepsilon_{1}}{\log \left(1+\varepsilon_{1}\right)}\right]
$$

où [.] désigne la partie entière. Alors

$$
\forall s>r, \forall k \geq 1, \quad \frac{d\left(k, N_{s}\right)}{N_{s}} \leq C \mu_{k}^{\left(N_{s}\right)} .
$$

On peut remarquer qu'une simple réécriture de la preuve de ce lemme permet de montrer (8) pour tout $N>N_{r}$. De plus cette relation entraîne que

(9) $\quad \forall s>r, \forall k \geq 1, \quad\left|\frac{d\left(k, N_{s}\right)}{N_{s}}-\mu_{k}^{\left(N_{s}\right)}\right| \leq(1 \vee(C-1)) \mu_{k}^{\left(N_{s}\right)}$.

Preuve (du Lemme B). Soit $k \geq 1$ fixé de façon quelconque. Pour $\bar{s}>r$, on considère $N_{\bar{s}}$ et on note $s_{*}=\bar{s}-r$. On définit encore

$$
\forall s \geq 1, \quad \mathcal{Q}_{s}=\left[N_{s-1}, N_{s}\left[\cup\left\{z \in \mathcal{P}^{*}: z \nmid k\right\} .\right.\right.
$$


Alors

$$
\forall s \geq 1, \quad \sum_{z \in \mathcal{Q}_{s}} \frac{1}{z}<C,
$$

où $C$ est une constante indépendante de $s$.

En effet, on a le résultat bien connu suivant (voir par exemple le théorème 428 dans $[\mathrm{HW}])$ :

$$
\forall x \geq 2, \quad \sum_{p \leq x, p \text { premier }} \frac{1}{p}=\log \log x+a+O\left(\frac{1}{\log x}\right),
$$

avec $a$ une constante absolue. Comme la suite $\left(N_{s}\right)_{s \geq 1}$ vérifie $N_{s+1} \leq N_{s}^{1+\varepsilon_{2}}$ on obtient

$$
\sum_{z \in \mathcal{Q}_{s}, z \text { premier }} \frac{1}{z}<C_{1} \quad(\text { constante indépendante de } s) .
$$

De plus

$$
\begin{aligned}
\sum_{z \in \mathcal{Q}_{s}, z \text { non premier }} \frac{1}{z} & \leq \sum_{p \text { premier }} \sum_{j \geq 2} \frac{1}{p^{j}} \\
& \leq \sum_{p \text { premier }} \frac{1}{p(p-1)} \leq 2 \sum_{n \geq 1} \frac{1}{n^{2}} \leq C_{2} .
\end{aligned}
$$

Majorons maintenant $d\left(k, N_{\bar{s}}\right) / N_{\bar{s}}$ :

$$
\begin{aligned}
\frac{d\left(k, N_{\bar{s}}\right)}{N_{\bar{s}}}= & \frac{1}{N_{\bar{s}}} \sum_{n=1}^{N_{\bar{s}}} \prod_{z \in \mathcal{P}^{*}, z \nmid k, z \leq N_{\bar{s}}}\left(1-\chi_{z}(n)\right) \quad \text { (d'après (5)) } \\
\leq & \frac{1}{N_{\bar{s}}} \sum_{n=1}^{N_{\bar{s}}} \prod_{s=1}^{s_{*}} \prod_{z \in \mathcal{Q}_{s}}\left(1-\chi_{z}(n)\right) \\
\leq & \frac{1}{N_{\bar{s}}} \sum_{n=1}^{N_{\bar{s}}} \prod_{s=1}^{s_{*}}\left\{\sum_{A \subset \mathcal{Q}_{s},|A|<k_{s}}(-1)^{|A|} \prod_{z \in A} \chi_{z}(n)\right. \\
& \left.+\sum_{A \subset \mathcal{Q}_{s},|A|=k_{s}} \prod_{z \in A} \chi_{z}(n)\right\},
\end{aligned}
$$

la dernière inégalité s'obtenant en appliquant le résultat du Lemme A pour $k_{s}=s_{*}-s+1$. Lorsqu'on estime le nombre de termes dans la somme du membre droit de l'inégalité on trouve que l'on a au plus $N_{\bar{s}}^{\alpha}$ termes de la forme $+\chi_{z}$ ou $-\chi_{z}$, avec (d'après notre choix pour $r$ )

$$
0<\alpha=\frac{1}{\varepsilon_{1}^{2}\left(1+\varepsilon_{1}\right)^{r-3}}<1 .
$$


En effet, le nombre de termes est inférieur à

$$
\begin{aligned}
\prod_{s=1}^{s_{*}} \sum_{k=1}^{k_{s}} C_{\left|\mathcal{Q}_{s}\right|}^{k} & \leq \prod_{s=1}^{s_{*}} \sum_{k=1}^{k_{s}}\left|\mathcal{Q}_{s}\right|^{k} \leq \prod_{s=1}^{s_{*}} \frac{\left|\mathcal{Q}_{s}\right|}{\left|\mathcal{Q}_{s}\right|-1}\left|\mathcal{Q}_{s}\right|^{k_{s}} \leq 2^{s_{*}} \prod_{s=1}^{s_{*}} N_{s}^{k_{s}} \\
& \leq \prod_{s=1}^{s_{*}} N_{s}^{k_{s}+1} \leq \prod_{s=1}^{s_{*}}\left(N_{s_{*}+r}^{\left(\frac{1}{1+\varepsilon_{1}}\right)^{s_{*}-s+r}}\right)^{k_{s}+1} \leq N_{s_{*}+r}^{\sigma},
\end{aligned}
$$

avec

$$
\sigma=\sum_{s=1}^{s_{*}}\left(k_{s}+1\right)\left(\frac{1}{1+\varepsilon_{1}}\right)^{s_{*}-s+r} .
$$

Or

$$
\begin{aligned}
\sigma & =\sum_{s=1}^{s_{*}}\left(k_{s}+1\right)\left(\frac{1}{1+\varepsilon_{1}}\right)^{s_{*}-s+r}=\left(\frac{1}{1+\varepsilon_{1}}\right)^{r-1} \sum_{u=1}^{s_{*}} \frac{u+1}{\left(1+\varepsilon_{1}\right)^{u}} \\
& \leq\left(\frac{1}{1+\varepsilon_{1}}\right)^{r-1} \sum_{u \geq 0} \frac{u+1}{\left(1+\varepsilon_{1}\right)^{u}} \\
& \leq \frac{1}{\varepsilon_{1}^{2}\left(1+\varepsilon_{1}\right)^{r-3}}=\alpha
\end{aligned}
$$

D'où le résultat.

De plus, on vérifie très facilement que

$$
\forall N \geq 1, \forall z \in \mathbb{Z}, \quad\left|\frac{1}{N} \sum_{n=1}^{N} \chi_{z}(n)-\frac{1}{z}\right| \leq \frac{1}{N} .
$$

Donc,

$$
\begin{aligned}
& \frac{d\left(k, N_{\bar{s}}\right)}{N_{\bar{s}}} \\
\leq & \prod_{s=1}^{s_{*}}\left\{\sum_{A \subset \mathcal{Q}_{s},|A|<k_{s}}(-1)^{|A|} \prod_{z \in A} \frac{1}{z}+\sum_{A \subset \mathcal{Q}_{s},|A|=k_{s}} \prod_{z \in A} \frac{1}{z}\right\}+N_{\bar{s}}^{\alpha-1} \\
\leq & \prod_{s=1}^{s_{*}}\left\{\prod_{z \in \mathcal{Q}_{s}}\left(1-\frac{1}{z}\right)+2 \sum_{A \subset \mathcal{Q}_{s},|A|=k_{s}} \prod_{z \in A} \frac{1}{z}\right\}+N_{\bar{s}}^{\alpha-1} \quad \text { (Lemme A) } \\
\leq & C \prod_{s=1}^{s_{*}} \prod_{z \in \mathcal{Q}_{s}}\left(1-\frac{1}{z}\right) \cdot \prod_{s=1}^{s_{*}}\left(1+\left(\frac{C^{\prime}}{k_{s}}\right)^{k_{s}}\right)+N_{\bar{s}}^{\alpha-1},
\end{aligned}
$$

car

$$
\sum_{A \subset \mathcal{Q}_{s},|A|=k_{s}} \prod_{z \in A} \frac{1}{z} \leq \frac{1}{k_{s} !}\left(\sum_{z \in \mathcal{Q}_{s}} \frac{1}{z}\right)^{k_{s}} \leq\left(\frac{C}{k_{s}}\right)^{k_{s}}
$$


Le premier produit dans le dernier terme de l'inégalité (12) est majoré par $C \mu_{k}^{\left(N_{\bar{s}}\right)}$, avec $C$ une constante ne dépendant que de $r$, et le second par $\prod_{s \geq 1}\left(1+\left(C^{\prime} / s\right)\right)^{s}$ qui est inférieur à une constante. D'où

$$
\frac{d\left(k, N_{\bar{s}}\right)}{N_{\bar{s}}} \leq C \mu_{k}^{\left(N_{\bar{s}}\right)}+N_{\bar{s}}^{\alpha-1} \leq C^{\prime} \mu_{k}^{\left(N_{\bar{s}}\right)},
$$

car

$$
\forall N \geq 1, \forall k \in \mathbb{Z}, \quad \mu_{k}^{(N)} \geq C \prod_{p \leq N, p \text { premier }}\left(1-\frac{1}{p}\right) \geq \frac{C^{\prime}}{\log N} .
$$

Ceci termine la preuve du Lemme B.

Nous allons maintenant estimer la différence $d\left(k, N_{\bar{s}}\right) / N_{\bar{s}}-\mu_{k}^{\left(N_{\bar{s}}\right)}$ de façon plus précise. On garde les mêmes notations que dans la preuve du Lemme B. D'après le Lemme A,

$$
\begin{array}{r}
\left|\prod_{s=1}^{s_{*}} \prod_{z \in \mathcal{Q}_{s}}\left(1-\chi_{z}\right)-\prod_{s=1}^{s_{*}-1} \prod_{z \in \mathcal{Q}_{s}}\left(1-\chi_{z}\right)\left\{\sum_{A \subset \mathcal{Q}_{s_{*}},|A|<k_{s_{*}}}(-1)^{|A|} \prod_{z \in A} \chi_{z}\right\}\right| \\
\leq \sum_{A \subset \mathcal{Q}_{s_{*}},|A|=k_{s_{*}}} \prod_{z \in A} \chi_{z} .
\end{array}
$$

En itérant ceci on obtient

$$
\begin{aligned}
& \left|\prod_{s=1}^{s_{*}} \prod_{z \in \mathcal{Q}_{s}}\left(1-\chi_{z}\right)-\prod_{s=1}^{s_{*}}\left\{\sum_{A \subset \mathcal{Q}_{s},|A|<k_{s}}(-1)^{|A|} \prod_{z \in A} \chi_{z}\right\}\right| \\
& \leq \sum_{t=1}^{s_{*}} \mid \prod_{s=1}^{s_{*}-t+1} \prod_{z \in \mathcal{Q}_{s}}\left(1-\chi_{z}\right) \prod_{s=s_{*}-t+2}^{s_{*}}\left\{\sum_{A \subset \mathcal{Q}_{s},|A|<k_{s}}(-1)^{|A|} \prod_{z \in A} \chi_{z}\right\} \\
& \quad-\prod_{s=1}^{s_{*}-t} \prod_{z \in \mathcal{Q}_{s}}\left(1-\chi_{z}\right) \prod_{s=s_{*}-t+1}^{s_{*}}\left\{\sum_{A \subset \mathcal{Q}_{s},|A|<k_{s}}(-1)^{|A|} \prod_{z \in A} \chi_{z}\right\} \mid \\
& \leq \sum_{t=1}^{s_{*}} \mid \prod_{z \in \mathcal{Q}_{s_{*}-t+1}}\left(1-\chi_{z}\right)-\sum_{A \subset \mathcal{Q}_{s_{*}-t+1},|A|<k_{s_{*}-t+1}}\left(-A\left|\prod_{z \in A} \chi_{z}\right|\right. \\
& \quad \times\left|\prod_{s=s_{*}-t+2}\left\{\sum_{A \subset \mathcal{Q}_{s},|A|<k_{s}}(-1)^{|A|} \prod_{z \in A} \chi_{z}\right\}\right| \\
& \leq \sum_{u \leq s_{*}}\left(\sum_{A \subset \mathcal{Q}_{u},|A|=k_{u}} \prod_{z \in A} \chi_{z}\right) \prod_{v=u+1}^{s_{*}}\left(1+\sum_{A \subset \mathcal{Q}_{v},|A|=k_{v}} \prod_{z \in A} \chi_{z}\right) .
\end{aligned}
$$

En sommant maintenant cette relation par rapport à $\left(1 / N_{\bar{s}}\right) \sum_{n=1}^{N_{\bar{s}}}$, et en 
remarquant que

$$
\forall N \geq 1, \forall z \in \mathbb{Z}, \quad \frac{1}{N} \sum_{n=1}^{N} \chi_{z}(n) \leq \frac{1}{z},
$$

on trouve

$$
\begin{aligned}
& \mid \frac{1}{N_{\bar{s}}} \sum_{n=1}^{N_{\bar{s}}} \prod_{s=1}^{s_{*}} \prod_{z \in \mathcal{Q}_{s}}\left(1-\chi_{z}(n)\right) \\
& \quad-\frac{1}{N_{\bar{s}}} \sum_{n=1}^{N_{\bar{s}}} \prod_{s=1}^{s_{*}} \sum_{A \subset \mathcal{Q}_{s},|A|<k_{s}}(-1)^{|A|} \prod_{z \in A} \chi_{z}(n) \mid \\
& \leq \sum_{u \leq s_{*}}\left(\sum_{A \subset \mathcal{Q}_{u},|A|=k_{u}} \prod_{z \in A} \frac{1}{z}\right) \prod_{v=u+1}^{s_{*}}\left(1+\sum_{A \subset \mathcal{Q}_{v},|A|=k_{v}} \prod_{z \in A} \frac{1}{z}\right) .
\end{aligned}
$$

De plus, lors de la démonstration du Lemme B, nous avons montré que le nombre de termes apparaissant dans de telles sommes est inférieur ou égal à $N_{\bar{s}}^{\alpha}$ avec $\alpha=1 /\left(\varepsilon_{1}^{2}\left(1+\varepsilon_{1}\right)^{r-3}\right)$, et que

$$
\forall N \geq 1, \forall z \in \mathbb{Z}, \quad\left|\frac{1}{N} \sum_{n=1}^{N} \chi_{z}(n)-\frac{1}{z}\right| \leq \frac{1}{N} .
$$

Ceci entraîne que

$$
\begin{aligned}
\mid \frac{1}{N_{\bar{s}}} \sum_{n=1}^{N_{\bar{s}}} \prod_{s=1}^{s_{*}}\left\{\sum_{A \subset \mathcal{Q}_{s},|A|<k_{s}}(-1)^{|A|} \prod_{z \in A} \chi_{z}(n)\right\} \\
\quad-\prod_{s=1}^{s_{*}}\left\{\sum_{A \subset \mathcal{Q}_{s},|A|<k_{s}}(-1)^{|A|} \prod_{z \in A} \frac{1}{z}\right\} \mid \leq N_{\bar{s}}^{\alpha-1} .
\end{aligned}
$$

Combinons alors les relations (14) et (15) par inégalité triangulaire, de sorte que

$$
\begin{aligned}
& \left|\frac{1}{N_{\bar{s}}} \sum_{n=1}^{N_{\bar{s}}} \prod_{s=1}^{s_{*}} \prod_{z \in \mathcal{Q}_{s}}\left(1-\chi_{z}(n)\right)-\prod_{s=1}^{s_{*}}\left\{\sum_{A \subset \mathcal{Q}_{s},|A|<k_{s}}(-1)^{|A|} \prod_{z \in A} \frac{1}{z}\right\}\right| \\
& \quad \leq \sum_{u \leq s_{*}}\left(\sum_{A \subset \mathcal{Q}_{u},|A|=k_{u}} \prod_{z \in A} \frac{1}{z}\right) \prod_{v=u+1}^{s_{*}}\left(1+\sum_{A \subset \mathcal{Q}_{v},|A|=k_{v}} \prod_{z \in A} \frac{1}{z}\right)+N_{\bar{s}}^{\alpha-1} .
\end{aligned}
$$

En appliquant une fois de plus le Lemme A à cette dernière inégalité, ainsi que la relation (13), nous obtenons

$$
\left|\frac{1}{N_{\bar{s}}} \sum_{n=1}^{N_{\bar{s}}} \prod_{s=1}^{s_{*}} \prod_{z \in \mathcal{Q}_{s}}\left(1-\chi_{z}(n)\right)-\prod_{s=1}^{s_{*}} \prod_{z \in \mathcal{Q}_{s}}\left(1-\frac{1}{z}\right)\right|
$$




$$
\begin{aligned}
& \leq 2 \sum_{u \leq s_{*}}\left[1+\left(\frac{C}{k_{u+1}}\right)^{k_{u+1}}\right] \ldots\left[1+\left(\frac{C}{k_{s_{*}}}\right)^{k_{s_{*}}}\right] \frac{1}{k_{u} !}\left(\sum_{z \in \mathcal{Q}_{u}} \frac{1}{z}\right)^{k_{u}}+N_{\bar{s}}^{\alpha-1} \\
& \leq C \sum_{u \leq s_{*}}\left(\prod_{v=u+1}^{s_{*}}\left(1+\left(\frac{C}{k_{v}}\right)^{k_{v}}\right)\right) \frac{\left(\sum_{z \in \mathcal{Q}_{u}} 1 / z\right)^{k_{u}}}{k_{u} !}+N_{\bar{s}}^{\alpha-1} \\
& \leq C \sum_{u \leq s_{*}} 4^{-\left(s_{*}-u\right)} \sum_{z \in \mathcal{Q}_{u}} \frac{1}{z}+N_{\bar{s}}^{\alpha-1} .
\end{aligned}
$$

A l'aide du Lemme A on montre encore que

$$
\begin{aligned}
\left|\frac{1}{N_{\bar{s}}} \sum_{n=1}^{N_{\bar{s}}} \prod_{z \in \mathcal{P}^{*}, z \nmid k}\left(1-\chi_{z}(n)\right)-\frac{1}{N_{\bar{s}}} \sum_{n=1}^{N_{\bar{s}}} \prod_{s=1}^{s_{*}} \prod_{z \in \mathcal{Q}_{s}}\left(1-\chi_{z}(n)\right)\right| \\
\leq \frac{1}{N_{\bar{s}}} \sum_{n=1}^{N_{\bar{s}}}\left|\prod_{s=s_{*}+1}^{\bar{s}} \prod_{z \in \mathcal{Q}_{s}}\left(1-\chi_{z}(n)\right)-1\right| \\
\leq \frac{1}{N_{\bar{s}}} \sum_{n=1}^{N_{\bar{s}}} \sum_{z \in \mathcal{Q}_{s_{*}+1} \cup \ldots \cup \mathcal{Q}_{\bar{s}}} \chi_{z}(n) \leq \sum_{z \in \mathcal{Q}_{s_{*}+1} \cup \ldots \cup \mathcal{Q}_{\bar{s}}} \frac{1}{z},
\end{aligned}
$$

et aussi que

$$
\begin{aligned}
\left|\mu_{k}^{\left(N_{\bar{s}}\right)}-\prod_{s=1}^{s_{*}} \prod_{z \in \mathcal{Q}_{s}}\left(1-\frac{1}{z}\right)\right| & \leq\left|\prod_{s=s_{*}+1}^{\bar{s}} \prod_{z \in \mathcal{Q}_{s}}\left(1-\frac{1}{z}\right)-1\right| \\
& \leq \sum_{z \in \mathcal{Q}_{s_{*}+1} \cup \ldots \cup \mathcal{Q}_{\bar{s}}} \frac{1}{z}
\end{aligned}
$$

En se rappelant que $s_{*}=\bar{s}-r$ et en regroupant les trois inégalités (16), (17) et (18), on obtient

$$
\left|\frac{d\left(k, N_{\bar{s}}\right)}{N_{\bar{s}}}-\mu_{k}^{\left(N_{\bar{s}}\right)}\right| \leq C \sum_{t \leq \bar{s}} 4^{-(\bar{s}-t)} \sum_{z \in \mathcal{Q}_{t}} \frac{1}{z}+N_{\bar{s}}^{\alpha-1} .
$$

D'où d'après (9) on a

$$
\left|\frac{d\left(k, N_{\bar{s}}\right)}{N_{\bar{s}}}-\mu_{k}^{\left(N_{\bar{s}}\right)}\right| \leq C \inf \left\{\sum_{t \leq \bar{s}} 4^{-(\bar{s}-t)} \sum_{z \in \mathcal{Q}_{t}} \frac{1}{z}, \mu_{k}^{\left(N_{\bar{s}}\right)}\right\}+N_{\bar{s}}^{\alpha-1} .
$$

Appliquons maintenant le lemme suivant, dont la démonstration est évidente.

Lemme C. Soient $\left(\alpha_{t}\right)$ et $\beta$ des réels positifs. Alors,

$$
\inf \left(\sum_{t} \alpha_{t}, \beta\right) \leq \sum_{t}\left(\alpha_{t} \beta\right)^{1 / 2} .
$$


Ce résultat entraîne que

$$
\begin{aligned}
\mid \frac{d\left(k, N_{\bar{s}}\right)}{N_{\bar{s}}} & -\mu_{k}^{\left(N_{\bar{s}}\right)} \mid \\
& \leq C \sum_{t \leq \bar{s}} 2^{-(\bar{s}-t)} \prod_{z \in \mathcal{Q}_{t^{\prime}}, t^{\prime}<t}\left(1-\frac{1}{z}\right)^{1 / 2} \cdot\left(\sum_{z \in \mathcal{Q}_{t}} \frac{1}{z}\right)^{1 / 2}+N_{\bar{s}}^{\alpha-1} .
\end{aligned}
$$

Donc

$$
\begin{aligned}
\sum_{s \geq r}\left|\frac{d\left(k, N_{s}\right)}{N_{s}}-\mu_{k}^{\left(N_{s}\right)}\right|^{2} \leq & C \sum_{s \geq 1} \sum_{t \leq s} 2^{-(\bar{s}-t)} \prod_{z \in \mathcal{Q}_{t^{\prime}}, t^{\prime}<t}\left(1-\frac{1}{z}\right) \cdot \sum_{z \in \mathcal{Q}_{t}} \frac{1}{z} \\
& +C^{\prime} \sum_{s \geq 1} \frac{1}{N_{s}^{2(1-\alpha)}} \\
\leq & C^{\prime \prime} \sum_{t \geq 1} \prod_{z \in \mathcal{Q}_{t^{\prime}}, t^{\prime}<t}\left(1-\frac{1}{z}\right) \cdot \sum_{z \in \mathcal{Q}_{t}} \frac{1}{z},
\end{aligned}
$$

qui est bornée par une constante absolue d'après (11). Ceci termine donc la preuve du théorème.

\section{Références}

[Bo] J. Bourgain, Problems of almost everywhere convergence related to harmonic analysis and number theory, Israel J. Math. 71 (1990), 97-127.

[HW] G. H. Hardy and E. M. Wright, An Introduction to the Theory of Numbers, 4th ed., Clarendon Press, Oxford, 1960.

[Ro] G. C. Rota, An "Alternierende Verfahren" for general positive operations, Bull. Amer. Math. Soc. 58 (1962), 95-102.

Université de Strasbourg

7, rue René Descartes

67084 Strasbourg Cedex, France

E-mail: Ruch@math.u-strasbg.fr 